This article was downloaded by: [Duke University Medical Center]

On: 21 May 2015, At: 09:48

Publisher: Routledge

Informa Ltd Registered in England and Wales Registered

Number: 1072954 Registered office: Mortimer House, 37-41

Mortimer Street, London W1T 3J H, UK

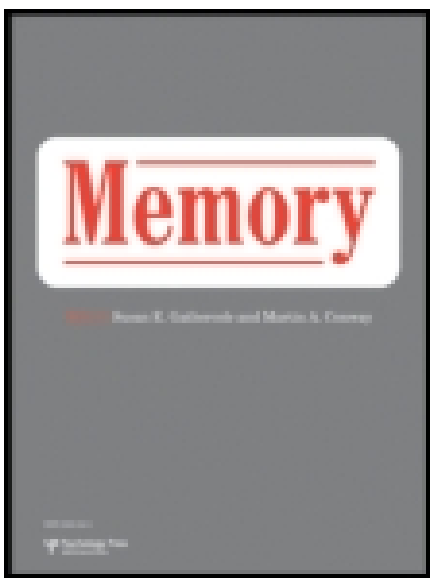

\title{
Memory
}

Publication details, including instructions for authors and subscription information:

http:// www.tandfonline.com/loi/ pmem20

\section{Spatial Imagery Preserves Temporal Order}

Maria E. Watson

Published online: 15 Oct 2010.

To cite this article: Maria E. Watson (1996) Spatial Imagery Preserves Temporal Order, Memory, 4:5, 515-534, DOI: 10.1080/ 741940777

To link to this article: http:// dx. doi.org/ 10.1080/ 741940777

\section{PLEASE SCROLL DOWN FOR ARTICLE}

Taylor \& Francis makes every effort to ensure the accuracy of all the information (the "Content") contained in the publications on our platform. However, Taylor \& Francis, our agents, and our licensors make no representations or warranties whatsoever as to the accuracy, completeness, or suitability for any purpose of the Content. Any opinions and views expressed in this publication are the opinions and views of the authors, and are not the views of or endorsed by Taylor \& Francis. The accuracy of the Content should not 
be relied upon and should be independently verified with primary sources of information. Taylor and Francis shall not be liable for any losses, actions, claims, proceedings, demands, costs, expenses, damages, and other liabilities whatsoever or howsoever caused arising directly or indirectly in connection with, in relation to or arising out of the use of the Content.

This article may be used for research, teaching, and private study purposes. Any substantial or systematic reproduction, redistribution, reselling, loan, sub-licensing, systematic supply, or distribution in any form to anyone is expressly forbidden. Terms \& Conditions of access and use can be found at http://www.tandfonline.com/page/terms-and-conditions 


\title{
Spatial Imagery Preserves Temporal Order
}

\author{
Maria E. Watson and David C. Rubin \\ Duke University, USA
}

\begin{abstract}
Line drawings were presented in either a spatial or a nonspatial format. Subjects recalled each of four sets of 24 items in serial order. Amount recalled in the correct serial order and sequencing errors were scored. In Experiment 1 items appeared either in consecutive locations of a matrix or in one central location. Subjects who saw the items in different locations made fewer sequencing errors than those who saw each item in a central location, but serial recall levels for these two conditions did not differ. When items appeared in nonconsecutive locations in Experiment 2, the advantage of the spatial presentation on sequencing errors disappeared. Experiment 3 included conditions in which both the consecutive and nonconsecutive spatial formats were paired with retrieval cues that either did or did not indicate the sequence of locations in which the items had appeared. Spatial imagery aided sequencing when, and only when, the order of locations in which the stimuli appeared could be reconstructed at retrieval.
\end{abstract}

\section{INTRODUCTION}

Behavioural and neuropsychological evidence indicates that visual imagery is not a unitary capacity but includes at least two largely independent systems. The behavioural evidence comes primarily from studies using the dual-task paradigm, a method that was first used in imagery studies to demonstrate a behavioural dissociation between imagery and verbal coding. More recently, this paradigm has been successfully adapted to dissociate visual and spatial components in imagery (Logie \& Marchetti, 1991; Tresch, Sinnamon, \& Seamon, 1993; see Logie, 1995, for a review). Evidence from neuropsychology indicates that these two imagery systems parallel two cortical visual systems, one devoted to object recognition, and the other to spatial perception (Farah, Hammond, Levine, \& Calvanio, 1988; Levine, Warach, \& Farah, 1985). Following the terminology for the cortical visual systems set forth by

Requests for reprints should be sent to David Rubin, Department of Experimental Psychology, Duke University, Durham, NC 27708-0086, USA.

This research was supported by a grant to the second author from the National Science Foundation BNS-90-10074. We would like to thank Kelly Braun, Robert Logie, Matt Schulkind, Jenny Zervakis, and anonymous reviewers for commenting on earlier versions of this manuscript, and Laura Anibal for assisting in data collection.

(C) 1996 Psychology Press, an imprint of Erlbaum (UK) Taylor \& Francis Ltd 
Ungerleider and Mishkin (1982), these imagery systems will be referred to as object and spatial imagery. We adopt the term system following Ungerleider and Mishkin (1982), Levine et al. (1985), and others, because there are two physically distinct neural pathways. Object imagery is used to represent the visual appearance of objects including colour and form, while spatial imagery is used to specify the location of an imaged object, and may also be involved in operations performed on the object representation, such as mental rotation and scanning (cf Farah, 1989). Object imagery, like object vision, is subserved by occipital-temporal cortex, while spatial imagery, like spatial vision, is subserved aby occipital-parietal cortex. For this reason, although imagery and perceptual Oideficits do not always occur together, impairments in object perception and Æobject imagery are commonly associated, as are impairments in spatial Iperception and spatial imagery, and object and spatial imagery may be

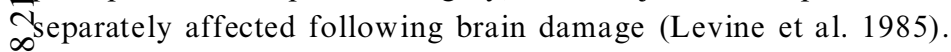

Two disclaimers must be made explicit. First, no neuroanatomical or Oneuropsychological measures are made here. We offer no direct tests about any $\mathrm{F}_{u}$ underlying neural substrate. Rather it is our reading of history that although there has long been evidence for an object and a spatial component to the visual

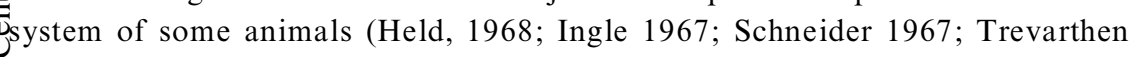
1968), until cognitive psychologists began considering the neural substrate of -imagery, visual imagery remained a unitary concept. We therefore base our $\sum_{2}^{\infty}$ object versus spatial division on neuropsychological data. Second, we attempt to make a clean behavioural-operational division between object and spatial id Zbehavioural and neural theories that accept this division assume that the two components are part of an integrated system.

The neuropsychological evidence for two imagery systems indicates that it is necessary to discriminate between the object and spatial systems in considering the mnemonic functions of imagery. However, in contrast to the many studies Zaimed at demonstrating the independence of object and spatial imagery, and T्.investigating the brain regions underlying these capacities, there has been little research directed at determining how each system aids memory. Most studies of the mnemonic effects of imagery, such as those reviewed by Paivio (1971), Apredate the common distinction between these two systems, so that conclusions are drawn about imagery as a unitary system. Assuming the distinction between object and spatial imagery, it is apparent that these earlier conclusions do not necessarily pertain to both systems. What is needed are studies that address the unique contribution of each system. Here we will focus on isolating the contribution of spatial imagery, concentrating on its function in preserving information about the order of items because this is a function typically not attributed to imagery. Our claim is not that spatial imagery is the only way to preserve order, but that it is one way. We therefore choose a manipulation that is as purely spatial as we can devise. 
Although there are few studies that address the function of spatial imagery, there is a separate body of literature on spatial memory. This literature indicates that spatial location is an important attribute of memory (Underwood, 1969) that may be encoded automatically (Andrade \& Meudell, 1993; Ellis 1990; Hasher \& Zacks, 1979) as well as effortfully. For example, recall for the location of information in a text is reliable even when subjects are not explicitly instructed to learn this information (Rothkopf, 1971; Zechmeister \& McKillip, 1972). Memory for the location of objects is more accurate than memory for the location of words with the same referents (Pezdek, Roman, \& Sobolik, 1986). In addition, Winograd and Church (1988) found that learning face-name associations is facilitated when the locations in which the faces appear remain constant. They propose that the observed facilitation is due not to strengthened face-name associations, but to the use of location as an added cue.

In attempting to define the role of spatial imagery, it is also instructive to consider imagery tasks involving both memory for location and memory for individual items, such as the method of loci, in which items to be remembered are imagined at particular points along a path. The critical question here concerns the mnemonic role of the locations, as memory for location is generally considered the function of the spatial system. It is not clear that pairing to-belearned items with distinct locations improves memory for the items, or even that the spatial component has a causal role. Three possibilities exist that will be separated experimentally here. One possibility is that the different locations simply provide unique settings in which to imagine the objects to be remembered, because in the real world spatial location and the scene at that location are confounded: if I look to the upper left I see a plant, if I look to the right I see a desk. That is, the visual detail at each location, rather than the spatial location itself, may serve as a memory cue. If this is the only benefit provided by spatial location then no advantage should be observed when the different locations do not differ in visual detail. Another possibility, suggested by Underwood (1969), is that interference between memories might be reduced to the extent that they are associated with different spatial locations. If this is the benefit provided by spatial location, then different spatial locations should help even if they are not in an easy-to-code sequential order. A third possibility is that the locations provide a sequencing function. This possibility was suggested by Cicero (in Yates, 1966, p.2) who, in a discussion of the method of loci in De oratore, stated that "the order of the places will preserve the order of the things" Underwood (1969, p.562 emphasis added) reiterates this hypothesis in commenting that when the method of loci was used by ancient orators, "The ideas of the speech ... were then associated with these focal points and ordered by the spatial relationships of the objects". If this is the benefit provided by spatial location then different locations that possess an easy-to-access order should improve sequencing. 
The function of sequencing information, however, has not traditionally been ascribed to mental imagery when imagery is considered as a unitary system. Healy (1977) argues that temporal order recall is mediated by a phonemic code, rather than by a visual code, as phonemic substitutions are observed, and interpolated vocalisation proves more detrimental than an interpolated spatial task. Paivio (1971) maintains that imagery is less suited for sequential memory than the verbal system, and that this is a consequence of inherent differences in the modalities with which these memory systems are allied. Imagery, like vision, is basically a parallel system; verbal memory, like audition and speech, is inherently sequential. Paivio (1975) illustrates the difference between the two तsystems with the following observation: in describing the layout of a room from Imemory, it is possible to begin from any one of many starting points. In contrast, $\sum_{\text {to }}$ recall verbal materials, such as a poem, it is easiest to begin at the beginning. तnformation from the middle of a poem is not easily accessed without first retrieving all that precedes it. According to Paivio then, a distinguishing feature Oof the imagery system is that there are no constraints on the order in which ت्ञnformation can be retrieved from an image.
Paivio (1971, p.180) also acknowledges that a sequential organisation can be mposed on the imagery representation, however:

\begin{abstract}
Imagery, therefore, is basically a parallel-processing system.... It is not specialized for serial processing unless linked to an integrated (symbolic) motor response system, such as might be involved in imaginally tracing the outline of a block letter ... or imagining oneself moving along a familiar route containing sequentially arranged "signposts," as advocated in one version of Simonides' technique of places and images.
\end{abstract}

Assuming the distinction between object and spatial imagery, it seems likely that the strengths of a parallel system derive from object imagery, and that gsequencing is accomplished using spatial imagery. If spatial imagery serves a क्षू order, independent of amount recalled. Therefore, although there may be no benefit of a spatial organisation on the overall amount of serial recall, even when Aspatial and temporal orders are redundant (Hitch \& Morton, 1975), an advantage may be observed with a more sensitive measure, such as scoring the number of sequencing errors. This contrast between total amount of serial recall and sequential ordering is common in memory research as a distinction between item and order information (e.g. Healy, 1974; Serra \& Nairne, 1993). Here it is used in the service of isolating the sequential aspect of spatial imagery: object imagery is measured by item information and spatial imagery by order information.

The experiments reported here isolate the contribution of spatial imagery to both the number of items recalled and to their serial order by presenting objects 
to be remembered either in a single location or in different locations of a matrix made out of identical squares. This procedure allows three possible roles of spatial imagery to be contrasted. First, the locations are not distinctive in terms of object qualities, so that if location functions only by providing distinctive visual cues then no benefit of the spatial presentation would be expected. Second, if the locations serve to reduce inter-item interference, then more items should be remembered when they are presented in different locations. Third, if the locations permit sequencing of the information to be learned, then presenting items in difficult locations should improve serial ordering, but would not necessarily increase amount recalled.

In Experiment 1, pictures of items to be remembered were presented either in a single location or in different consecutive locations. Serial recall instructions were used, and both serial recall and sequencing errors were scored. A second factor in the experiment was presentation rate. Subjects saw each list of items either at a rate of three seconds per item, or they saw each list presented three times in succession at a rate of one second per item. The use of two presentation rates was largely exploratory: it was included here to determine whether the effects of the spatial manipulation would emerge only under a distributed presentation, or would generalise to both single and distributed presentation conditions. It seemed likely that the slower presentation rate would facilitate the formation of an object image, while the faster, repeated presentation rate would preclude or hinder the formation of a detailed object image, but emphasise the sequence of locations. To anticipate the results, however, there were no interactions between presentation rate and presentation format. The results of the two presentation rates are therefore reported primarily as a replication. Experiment 2 further investigated the condition under which spatial location aids memory by presenting the items in random, rather than consecutive, locations. This was done to determine whether the mnemonic benefits of presenting items in different locations require that the spatial and temporal orders are redundant. Experiment 3 extended the findings of the earlier experiments by including a condition in which items appear in a random sequence of locations but some subjects are reminded of the sequence of locations at retrieval. This manipulation was intended to investigate whether a known sequence of random locations would permit sequencing.

\section{EXPERIMENT 1}

\section{Method}

Subjects. The 56 Duke undergraduate students, who participated to fulfil a course requirement, were run in small groups. Presentation format and set order conditions were run in a pseudo-random order. 
Stimuli. Subjects were shown four sets of 24 pictures previously normed to have one name (Snodgrass \& Vanderwart, 1980). Each picture was photographed in two different settings. For presentation in the spatial condition, each picture was photographed in one of the cells of a $6 \times 4$ matrix. Each set included one picture in each of the 24 matrix cells. For the nonspatial condition, each picture was photographed surrounded by a square frame of the same dimensions as one of the matrix cells. For the spatial presentation, items were individually presented in successive cells of the matrix. To maximise the distinctiveness of the spatial locations, the $6 \times 4$ matrix was divided into four $3 \times 2$ matrices and the order of presentation was left to right and top to bottom Nin each of these four quadrants, as depicted in Fig. 1. The projected matrix was 㞼pproximately $1.40 \mathrm{~m}$ high $\times 2.08 \mathrm{~m}$ wide, with each cell $0.34 \mathrm{~m} \times 0.34 \mathrm{~m}$. For $\sum_{\text {the }}$ single location presentation, each item appeared in one cell in a central $\bar{N}_{\infty}$ location. Subjects viewed the stimuli from distances ranging from $2.75 \mathrm{~m}$ to $+5.25 \mathrm{~m}$. The order of items within each set was identical for the matrix and single Olocation formats. Figure 1 depicts the first four items of a set in the spatial and

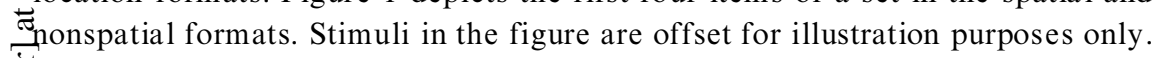
可In the experiment each matrix and each single location stimulus was presented ङुin the same location.

Apparatus. Slides were presented using a Kodak carousel $850 \mathrm{H}$ projector connected to a timing mechanism.

Design and Procedure. Half the subjects were assigned to the spatial condition, and half were assigned to the single location condition. Within each condition, subjects received one of two orders of the four lists of 24 slides. In Shis experiment and all others, no item appeared on more than one trial. Half of

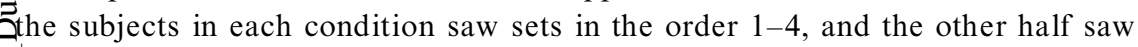
them in reverse order. Half of the subjects saw each set presented once at a 3zsecond rate, and half saw each set presented three times in succession at a 1E్ variables.

Subjects were instructed to try to remember the pictures by silently naming aeach item and forming a clear image of it. They were informed that they would later be asked to recall the items in serial order. After each set had been presented subjects worked on a Thurstone letter completion task (Simon \& Kotovsky, 1963) for 30 seconds. This task consists of letter series, each constructed according to a different rule. The subject's task is to deduce the rule and fill in the next letter in the series. Two of the easier problems used are $\mathrm{c} \mathrm{d} \mathrm{c} \mathrm{d}$ $\mathrm{c} \mathrm{d}_{-}$, and a a a b b b c c c d d . They were then given a minute and a half to write down as many items a they could recall in serial order. 


\section{Results}

Each recall protocol was scored for both serial recall and sequencing errors. Because subjects were instructed to list the items in order, serial recall was scored rather than the number of items recalled regardless of order. Serial recall was scored by omitting the minimum number of items necessary to leave the remaining items in correct order. To illustrate the manner in which the data were scored, imagine that the first 24 letters of the alphabet were shown in order as the 24 pictures. Suppose that a subject recalled 15 items in the following order: A, B, C, E, D, H, I, K, J, O, P, T, S, V, W. In this example, omitting the letters E, $\underset{\mathrm{N}}{\mathrm{K}}$, and $\mathrm{T}$ leaves the remaining items in correct serial order. Alternatively, the lletters D, J, and S could have been removed. In either case, however, three items $\sum$ must be omitted to leave the remaining items in correct order. The number of -items in correct serial order, in this case 12 , is the subject's serial recall score. The number of items that are out of order, i.e. that have to be omitted to leave githe remaining items in correct serial order, provides a measure of sequencing Ferrors. In this example, the number of sequencing errors is three. Because the Enumber of sequencing errors is limited by the total number of items recalled, a gnormalised measure of sequencing errors was used. The measure is a ratio of the

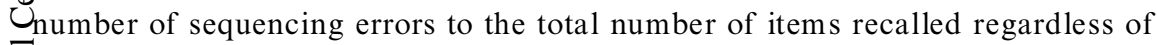
JुJorder. In the alphabetic example in which the subject recalled a total of 15 items eand made three sequencing errors, the percentage of sequencing errors is $3 / 15$ or $\Sigma_{20 \%}$. Omissions in recall, such as failing to report the letter $\mathrm{G}$ in this example, . Dsecond instance was ignored. Synonyms and responses that named items Evisually similar to the target were scored as correct, e.g. "bracelet" for chain, or "squirrel", for skunk.

Serial Recall. Mean serial recall scores are shown in the first two columns Zof Table 1. Serial recall was analysed using a mixed 2 (presentation format) $\times 2$ Z(presentation rate) $\times 2$ (set order) $\times 4$ (trial) ANOVA with trial as a repeated Iffactor. Presentation format did not affect amount recalled, $[F(1,48)=0.01$, $P=0.91]$, but there was a significant main effect of presentation rate, $\left[F(1,48)=5.29, \mathrm{MS}_{\mathrm{e}}=27.04, P<0.05\right]$, with three presentations at one second each being better than one presentation at three seconds. There was also a trial $\times$ set order interaction, $\left[F(3,144)=6.20, \mathrm{MS}_{\mathrm{e}}=7.02, P<0.0005\right]$, indicating that at least one of the four sets was either easier or more difficult to recall.

Sequencing Errors. Mean normalised sequencing errors are shown in columns three and four of Table 1 . Sequencing errors were analysed using a mixed 2 (presentation format) $\times 2$ (presentation rate) $\times 2$ (set order) $\times 4$ (trial) ANOVA with trial as a repeated factor. There was a main effect of presentation format, $\left[F(1,48)=19.79, \mathrm{MS}_{\mathrm{e}}=370.02, P<0.0001\right]$. No other main effects or interactions reached significance. 
TABLE 1

Experiment 1

\begin{tabular}{lcccccc}
\hline & \multicolumn{3}{c}{ Serial Recall } & \multicolumn{3}{c}{$\%$ Sequencing Errors } \\
\cline { 2 - 7 } Rate & Matrix & Single & Mean & Matrix & Single & Mean \\
\hline 1-second & $9.45(0.87)$ & $10.02(0.72)$ & $9.73(0.56)$ & $7.03(1.63)$ & $18.02(2.36)$ & $12.53(1.76)$ \\
3-second & $8.34(0.51)$ & $7.93(0.62)$ & $8.13(0.39)$ & $9.45(3.34)$ & $21.33(2.61)$ & $15.39(2.37)$ \\
Mean & $8.89(0.51)$ & $8.97(0.51)$ & & $8.24(1.84)$ & $19.67(1.75)$ & \\
\hline
\end{tabular}

Mean serial recall and percentage of sequencing errors for conditions in Experiment 1 (standard errors in parentheses).

\section{Discussion}

Spatial location could serve three functions: it could enhance memory by (1) only providing visually distinctive cues, (2) reducing interference between items and thereby increasing the number of items recalled, or (3) helping to preserve the sequence of the presented items. Our data suggest that first, spatial location is beneficial to order information even when the locations used are not distinctive in terms of visual detail. Second, presenting visual stimuli in a spatial array does not increase serial recall relative to presenting them in a single location but, third, it does reduce the number of sequencing errors by more than a factor of two. Moreover, this effect obtains both when the stimuli appear once for a period of three seconds and when they appear three times for one second apiece. Presenting items in consecutive spatial locations may preserve temporal information because spatial and temporal order are perfectly correlated, permitting knowledge of the spatial locations to be translated into knowledge regarding temporal order. This in turn suggests that the effects of providing a spatial organisation depend on whether subjects are able to remember the order of locations in which the items appeared.

\section{EXPERIMENT 2}

Experiment 2 was designed to determine whether presenting items in different spatial locations would improve sequencing performance when the locations in which the items appear are not consecutive, and therefore not easily learned. If a nonconsecutive series of locations fails to improve sequencing relative to nonspatial presentation format, this would suggest that the benefit of spatial organisation accrues only when the sequence of locations is known at recall. The procedure was identical to that used in Experiment 1, except that items in the spatial format were presented in non sequential cells of the matrix, and a different random sequence of locations was used for each of the four sets. 


\section{Method}

Subjects. Fifty-six Duke undergraduate students participated to fulfil a course requirement. No subject had participated in Experiment 1. Subjects were assigned to conditions based on the order in which they signed up to participate, and were run in small groups. As in Experiment 1, presentation format and set order conditions were run in a pseudo-random order.

Stimuli. The four sets of slides used in Experiment 1 served as stimuli. W ithin each set, however, the slides were randomised without replacement with तthe restriction that in the spatial condition the items would appear in 吾onconsecutive cells of the matrix. The order in which the items were presented $\sum_{w a s}$ the same for both spatial and single location formats.

Design and Procedure. The procedure was the same as that used in Experiment 1. Both the one-second and three-second presentation rates were Insed. Within each of these conditions, half of the subjects saw the items within a 荘 येंsingle matrix cell. As before, two set orders were used, one being the reverse of the other.

\section{Results}

The results of Experiment 2 are shown in Table 2.

Serial Recall. The data were analysed using a mixed 2 (presentation Fformat) $\times 2$ (presentation rate) $\times 2$ (set order) $\times 4$ (trial) ANOVA with trial as ${ }^{\text {a }}$ repeated factor. As in Experiment 1 , more items were recalled at the one$\rightarrow$ second presentation rate than at the three-second rate, $[F(1,48)=4.84$, $\left.\mathrm{MS}_{\mathrm{e}}=52.29, P<0.05\right]$. This effect was qualified by a higher order presentation Trate $\times$ trial $X$ set order interaction, $\left.F(3,144)=3.90, \mathrm{MS}_{\mathrm{e}}=5.56, P<0.05\right]$, indicating that the relative difficulty of the four sets varied with the rate of presentation. There was no effect of presentation format on serial recall, ๑[ $F(1,48)=1.23, P=0.27]$.

Sequencing Errors. A second analysis was done with sequencing errors as the dependent measure. This analysis yielded a main effect of presentation rate, $\left[F(1,48)=7.58, \mathrm{MS}_{\mathrm{e}}=839.50, P<0.01\right]$. Subjects who received three presentations at the one-second rate made fewer sequencing errors. A main effect of trial occurred, $\left[F(3,144)=2.88, \mathrm{MS}_{\mathrm{e}}=137.86, P<0.05\right]$. This effect was qualified by a higher order interaction of presentation rate $\times$ trial $\times$ set order, $\left[F(3,144)=3.61, \mathrm{MS}_{\mathrm{e}}=0.01, P<0.05\right]$, again indicating that the relative difficulty of the four sets varied with the presentation rate. In contrast to 
TABLE 2

Experiment 2

\begin{tabular}{lcccccc}
\hline & \multicolumn{3}{c}{ Serial Recall } & \multicolumn{3}{c}{$\%$ Sequencing Errors } \\
\cline { 2 - 7 } Rate & Matrix & Single & Mean & Matrix & Single & Mean \\
\hline 1-second & $8.73(0.62)$ & $11.39(0.78)$ & $10.06(0.55)$ & $24.97(3.79)$ & $14.48(2.59)$ & $19.73(2.47)$ \\
3-second & $8.20(1.31)$ & $7.68(0.95)$ & $7.94(0.80)$ & $29.05(5.03)$ & $31.72(3.74)$ & $30.39(3.09)$ \\
Mean & $8.46(0.71)$ & $9.54(0.70)$ & & $27.01(3.12)$ & $23.10(2.78)$ & \\
\hline
\end{tabular}

Mean serial recall and percentage of sequencing errors for conditions in Experiment 2 (standard errors in parentheses).

Experiment 1, however, there was no difference between the two presentation formats in the proportion of items recalled out of sequence.

\section{Discussion}

The findings of Experiment 2 indicate that the smaller proportion of sequencing errors associated with the spatial presentation in Experiment 1 does not obtain when the locations in which the items appear are nonsequential. These results suggest that the spatial organisation is only beneficial when the series of locations can be reconstructed at the time of retrieval. In other words, it is plausible that the random sequence does not confer an advantage because the sequence of locations itself is not easily learned. As a result, subjects could not recall the sequence of locations in which the stimuli appeared, and were therefore unable to use this information to remember the order in which the items appeared.

A slightly different way of framing the hypothesis is to suggest that a sequence of locations can become encoded in memory as a spatial pathway, and that this pathway can be used to remember a sequence of events. That is, spatial imagery helps sequencing only when the relation between spatial and sequential order is known at retrieval. According to this view, the consecutive locations used in Experiment 1 are effective because they constitute an easily learned pathway. To further explore the plausibility of this idea, Experiment 3 included retrieval cues that could be used to indicate the sequence of locations in which the stimuli appeared. By pairing such cues with a random-spatial presentation the learning of a spatial pathway can be simulated.

\section{EXPERIMENT 3}

Experiment 3 replicates and extends the findings of the first two experiments. The sequential-spatial, random-spatial, and single location presentation formats are used again, but here all in the same experiment as a check on our earlier work. In addition, we added retrieval cues in the form of arrows to all 
conditions. In one condition of the random-spatial presentation format these cues indicated the order of locations in which the items appeared, thus providing a pathway at recall when none was inherent at encoding. To control for any possible detrimental effects of the arrow cues we added them to all conditions.

In all conditions, subjects wrote down the name of a single item each time an arrow retrieval cue was shown. The first retrieval cue therefore indicated that the subject was to recall the first item, the second cue indicated that the subject was to recall the second item, and so forth. Retrieval cues were of two types: (1) spatial cues in which the arrows were used to indicate the order of locations in Which the stimuli had appeared, and (2) single location cues in which each arrow तpointed to the same central location.

Five conditions were used in Experiment 3. Each condition represented a Sarticular combination of presentation format (single location, random-spatial, خor sequential-spatial) and retrieval cue (spatial or single location). There are five instead of six conditions because in the single location condition all stimuli and Gretrieval cues were shown in a single central location, making the single location $\vec{F}_{\text {and }}$ spatial location retrieval cues identical. For the sequential-spatial condition 运with spatial cues, both stimuli and arrow cues were displayed in sequential cells 己ुof the matrix. For the random-spatial condition with spatial cues, the arrows Twere displayed in the same random sequence of locations as that in which the Ststimuli appeared. The sequential-spatial and random-spatial presentation formats $\sum^{\infty}$ were also each paired with single location cues. In these two conditions each zarrow appeared in the same central location, thereby providing no information rogarding the sequence of locations in which the stimuli had appeared, but . serving merely to cue the subject to write down the name of the next item. A Different sequence of locations was used for each of the four sets in the randomOspatial conditions.

The following predictions follow from the claim that spatial imagery will aid in the ordering of recalled items to the extent that there are separate spatial flocations at encoding that fall on a path that can be easily accessed at recall. T्. Comparisons between the single location format and both the sequential-spatial and random-spatial formats with single location retrieval cues should replicate the findings of Experiments 1 and 2. As in Experiment 1, sequencing errors Ashould be significantly lower when stimuli appear in the sequential-spatial format than when they appear in a single location. Because a consecutive order of locations is easily learned, the provision of spatial cues following the sequential-spatial presentation should not produce a noticeable improvement in sequencing compared to the single location cue condition. That is, no significant difference between the spatial and nonspatial cue conditions is expected when stimuli appear in a sequential-spatial format. A random-spatial format with single location cues should provide no advantage in sequencing over a single location, as in Experiment 2. However, when a random-spatial presentation is paired with spatial retrieval cues, sequencing errors should be lower than when 
all stimuli appear in the same location because a spatial presentation format should be beneficial to the extent that the sequence of locations in which the items appeared can be reconstructed at the time of retrieval.

\section{Methods}

Subjects. Eighty undergraduate students participated to fulfil a course requirement. No subject had participated in the earlier experiments. Subjects were run in small groups. Groups were assigned to conditions in a pseudorandom order.

Stimuli. The stimulus items were the same as those used in Experiments 1 and 2, however for this experiment the four sets were reconstituted by randomising the four slides for each of the 24 serial positions. The order in which the items were presented in the single location condition was the same as that used for the two sequential-spatial conditions.

Slides of the nonspatial retrieval cues were constructed by photographing an arrow pointing to a square frame, identical to that which surrounded each of the stimuli in the single location conditions. Slides of the spatial retrieval cues were constructed by taking 24 photographs, each of which included the matrix with an arrow pointing to one of the matrix cells. Figure 1 shows the first four single location retrieval cues and the first four retrieval cues for the sequential-spatial condition with spatial cues. The matrices and single location frames are offset only for purposes of illustration.

The stimuli were projected using a Kodak projector. The projected matrix was $1.14 \mathrm{~m}$ high $\times 1.70 \mathrm{~m}$ wide. The dimensions of the single frame were $28 \mathrm{~cm} \times 28 \mathrm{~cm}$. Subjects viewed the stimuli from distances ranging from $1.45 \mathrm{~m}$ to $4.14 \mathrm{~m}$.

Procedure. Stimuli were shown for one second apiece, with each set shown three times. The three-second rate was not included as no significant interactions between format and rate were found in the first two experiments. After the third presentation, subjects worked on a Thurstone letter completion task for 30 seconds. Subjects were then shown the 24 arrow cues at a constant rate of about one every five seconds. Subjects in all conditions were told that they would be asked to recall the items in the order in which they were presented. For the two conditions in which spatial cues were provided, subjects were informed that the sequence of locations in which arrow cues appeared would be the same as the sequence in which the stimuli appeared, and that each time they saw an arrow cue they were to write down the name of the item that appeared in that location. For the conditions in which nonspatial cues were provided, subjects were instructed to write the name of the first item when the first cue appeared, the second item when the second cue appeared and so forth. All subjects were 
instructed to write down only one item for each arrow, and to put an " $\mathrm{X}$ " on the line if they could not recall an item. Two orders of set presentation were used, as in the earlier experiments. Presentation format and set order served as betweensubjects variables.

\section{Results}

Eleven subjects did not recall any items in the first set. As this was thought to reflect a failure to understand the instructions, set 1 was retroactively designated ${ }^{2}$ practice set and excluded from all analyses. One subject who did not recall any Nitems from sets 1 and 2 was replaced. Recall protocols were scored in the same Imanner as in Experiments 1 and 2, except that all instances of items written $\Sigma_{\text {more than once were not scored. This change in the scoring procedure was }}$ $\overbrace{\infty}$ necessary because a few subjects adopted the strategy of filling most of the fresponse sheet with only a few items. The results of Experiment 3 are shown in OेTable 3.

Serial Recall. Serial recall was analysed using a mixed 5 (presentation format/retrieval cue combination) $\times 2$ (set order) $\times 3$ (trial) ANOVA with trial a a repeated factor. There was a main effect of trial, $[F(2,140)=7.44$, $\left.\mathrm{MS}_{\mathrm{e}}=5.64, \quad P<0.001\right]$. The means for trials $2-4$ were, respectively: 8.28 $(\mathrm{SE}=0.37), 9.43(\mathrm{SE}=0.47)$ and $9.61(\mathrm{SE}=0.45)$. There was also a main effect tof the presentation format/retrieval cue combination, $[F(4,70)=3.88$, MS $\left.\mathrm{MS}_{\mathrm{e}}=29.64, P<0.01\right]$. Tukey's HSD test performed on the mean serial recall s scores for the five conditions indicated that serial recall in the two sequentialSpatial conditions was higher than in the condition in which a random-spatial format was paired with spatial cues.

Sequencing Errors. Two analyses of sequencing errors were performed. Jirst, a single planned comparison was used to test the predicted ordering of the T्ञfive conditions in the proportion of sequencing errors. The tested prediction was as follows: performance is expected to be lowest with the single location format and random-spatial format with single location cues because there is no pathway @information in these conditions; no difference between these conditions was expected. Performance should be highest in the two sequential spatial conditions; no difference between these conditions was expected because, since the left to right, top to bottom order is easily learned, the arrow cues provide no additional information. Finally, sequencing errors should be at an intermediate level when the random-spatial array is paired with spatial cues because the arrows would help provide a pathway, although one less useful than that given by a predictable spatial organisation. The test for this ordering, $-1,-1,0,1,1$, was significant, $\left[F(1,70)=29.43, \mathrm{MS}_{\mathrm{e}}=118.02, P<0.0001\right]$. A second contrast was performed to test for a nonlinear component in this ordering by comparing 
TABLE 3

Experiment 3

$\%$ Sequencing Errors

Single location/single location cues

Random-spatial/single location cues

Random-spatial/spatial cues

Sequential-spatial/spatial cues

Sequential-spatial/single location cues
$9.40(0.91)$
$7.92(0.77)$
$7.13(0.60)$
$10.40(0.67)$
$10.69(0.83)$
$19.99(3.13)$
$19.60(3.97)$
$13.40(2.56)$
$5.52(1.56)$
4.60 (1.11)

Mean serial recall and percentage of sequencing errors for conditions in Experiment 3 (standard errors in parentheses).

the mean of the random-spatial presentation with spatial cues condition to the average of the four remaining conditions. As expected, this -1 , $-1,4,-1,-1$ contrast was not significant $[F(1,70)=0.10, P=0.75]$. The sequencing results do not change if trial 1 is included in the analyses, with the average proportion of sequencing errors of those subjects who failed to respond on trial 1 calculated from the remaining three trials. The contrast for the ordering of the five means remains significant, $\left[F(1,70)=40.95, \mathrm{MS}_{\mathrm{e}}=0.01, P<0.0001\right]$, and the second contrast to test for a nonlinear component in the ordering remains nonsignificant, $[F(1,70)=0.20, P=0.65]$.

\section{Discussion}

Experiment 3 replicated the finding of Experiment 1 that a lower proportion of sequencing errors is made when items to be remembered are displayed in consecutive locations. It replicated the finding of Experiment 2 that the benefit of the spatial organisation is abolished when the order of locations is nonconsecutive. These earlier findings replicated despite the possibility that the use of the arrow cues added an effortful component to the processing of spatial information. It was hypothesised that in order to benefit from the spatial presentation, the sequence of locations itself must be learned. Therefore, with no prior training, only a consecutive sequence is effective. On this interpretation, the difference between the sequential-spatial and random-spatial formats is due to subjects' inability to reconstruct the random order of locations at the time of retrieval. The prediction that cues to the order of locations would allow subjects to benefit from the random-spatial presentation received support in the present study. The disparity between the sequential-spatial and random-spatial conditions may also result from differences in encoding not only the locations, but also the pictures themselves. For example, subjects may rely exclusively on verbal rehearsal in the random-spatial conditions. In addition, the need to shift attention unpredictably in the random-spatial conditions may increase processing demands and simultaneously decrease processing time per item. Such 
differences in encoding may help to explain why serial recall was also higher when items were presented in sequential locations than when they were presented in random locations.

\section{GENERAL DISCUSSION}

The studies presented here demonstrate that spatial imagery serves a memory function that is distinct from, and complementary to, that served by object imagery, namely, that of sequencing events in memory. The effect of the spatial Opresentation is robust: the difference between the spatial and nonspatial Oppresentations is greater than 2:1 for sequencing errors in Experiment 1, and Igreater than 3:1 in Experiment 3. Our results are consistent with the findings from studies of mnemonic systems and of selective interference; findings that خhelp to clarify the role spatial imagery plays in the preservation of order information. We consider the studies of mnemonic systems first.

Roediger (1980) compared immediate and delayed recall of 20-word lists using either an elaborative rehearsal strategy or one of four imagery strategies,

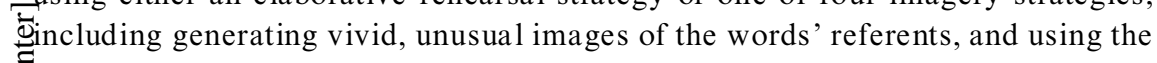
Ümethod of loci. Subjects could recall the items in any order, but were asked to a assign the words to the correct serial position. Roediger found that although the - method of loci produced superior recall to the imagery strategy with both $\sum^{\infty}$ unordered recall and ordered recall scoring, the advantage given by the loci mnemonic was greater when order was taken into account. The pegword mnemonic and link method were also effective in ordering recall, although the Zlink method was less effective than the other two mnemonics in the immediate Drecall test.

Both the pegword and loci mnemonics involve generating interactive images, and therefore appear to have roughly equivalent contributions from the object $>$ system. They differ, however, in the manner in which sequencing is preserved. Jn the pegword mnemonic the numbers one to ten are each associated with a Tु్thyming peg, e.g. one-bun, two-shoe, three-tree and so on. Each word to be learned is then paired with the pegword and an interactive image of the referents Zof these two words is constructed. For example, if the first word is "mouse", ๑one might imagine a mouse gnawing on a hamburger bun. The target words are later retrieved in serial order by using the rhyme to recall the pegword, and then retrieving the image of the peg and target item.

Like the pegword mnemonic, the method of loci involves the construction of interactive images, and one function of the locations is to supply these object images. In addition, the locations also seem to fulfil the role served by the numbers in the pegword mnemonic, i.e. to permit the stimulus images to be retrieved in sequential order. In this way the locations, like the numbers, function to organise retrieval of the stimulus images, thereby increasing the likelihood that none is forgotten. This hypothesis regarding the function of 
spatial imagery is similar to one suggested by Paivio (1971), namely to keep track of what has and has not been accessed.

As the pegword mnemonic demonstrates, associating items with an ordered series of locations is not the only means of improving memory for sequence. For example, maintaining serial order has traditionally been acknowledged as a strength of the verbal system. Imagery, in contrast, has been characterised as illsuited to preserving order information. The results presented here emphasise the role of spatial imagery in sequencing, to underscore the contrast between the historical characterisation of imagery as a single system poorly suited to sequencing, and the characterisation of imagery as having separate visual and spatial components, with the latter adept at sequencing information.

Our observations of a classroom demonstration of the method of loci conducted by the second author (Rubin, 1995) also support this interpretation. In this demonstration, the locations used by the instructor were photographed. Following a demonstration of the effectiveness of the method, students were shown the series of slides, and the instructor described in detail how he imagined each item interacting with that particular setting. The instructor then presented the slides a second time in the same order, and asked the students for an incidental recall. The students report recalling over $80 \%$ correct despite the fact that, for them, the series of locations depicted in the slides did not constitute a known pathway. In contrast to Experiments 1 to 3, which provide a spatial component but omit the object component, this demonstration hints at the results of omitting the spatial component while providing an ordered set of retrieval cues that leaves the object component intact. That the omission of the spatial component does not seem to impair performance is consistent with our suggestion that the function of the series of locations is not to increase amount recalled, but to order recall, and secondly to organise retrieval of the object images.

If the sequential processing given by the spatial system permits more efficient retrieval (cf Paivio, 1971), it is surprising that, in Experiment 1, serial recall is not improved by the spatial presentation. Equivalent serial recall for spatial and nonspatial presentation formats is also reported by Hitch and Morton (1975). They do not report a sequencing measure and it is possible that, if they did, it would be consistent with our results. In the present study, however, it is important to qualify the conclusion regarding the absence of effects on serial recall by noting that if item order is disregarded, i.e. if the recall protocols were scored as though subjects had been given free recall instructions, there is a tendency in all three experiments for subjects in the single location condition to recall more items than subjects in the spatial condition. It is possible that redirecting attention to a new location for each item creates additional attentional demands or reduces processing time per item in the spatial condition. These effects may obscure the advantage of the spatial organisation, so that on a serial recall measure the performance of the two groups is equivalent. 
The results reported here also converge with findings from selective interference. For example, a nonvisual spatial task interferes with some types of judgements concerning the relative order of the months of the year. Specifically, compared to counting, a spatial task interferes with subjects' ability to determine which of two months is closer to a reference month, going backwards in time. In contrast, counting is more disruptive when subjects are asked to name the month that comes two, three, or four months after the reference month (Friedman, 1983). These results support Friedman's interpretation that whereas the "forwards" task is mediated by verbal memory, the "n. backwards" version relies on imagery. Friedman's experimental finding was Imade independently of a remarkably similar clinical observation by Critchley ( 1953, p.352) who wrote:

Most interesting and complicated of all are those spatial disorders which also involve the conception of time, or order, or sequence. Thus, at the simplest level, is the patient who is able to count, but not backwards: to recite the days of the week (months of the year, or letters of the alphabet), but not in reverse order.... A less obvious defect is shown by the patients who cannot visualize in correct order a list of monarchs or prime ministers; who mix up the sequence of historical events.
\end{abstract}

Both Critchley's observation and Friedman's finding suggest that subjects sometimes reason about temporal information by translating it into a spatial ¿representation. That translation seems essentially a reversal of what subjects Zwere able to do when spatial and temporal order were correlated in Experiment क्षे 1 , namely to convert information about the spatial locations in which the items Zappeared into knowledge of the temporal order in which they were presented. SMoreover, Critchley's general observation that a disturbance in temporal Sinformation sometimes occurs following parietal damage is consistent with the قُ role of spatial imagery suggested in this paper. Although it is impossible to make zany claims regarding the brain areas activated in the experiments reported here, gspatial imagery is generally thought to rely, at least in part, on parietal cortex. T्ञThough clearly speculative, it is nevertheless possible that if spatial imagery supports sequencing, then not only spatial deficits, but also certain sequencing deficits would be associated with damage to parietal cortex.

The sequencing function of spatial imagery is also suggested by the nature of the spatial tasks used in two recent interference studies. In experiments by both Logie and Marchetti (1991) and Smyth and Scholey (1994) the spatial tasks were not simply memory for fixed locations, but rather memory for location sequences. Logie and Marchetti note that in a spatial task like Brooks' matrix task, in which subjects listen to and then recall a series of spatial directions that describe a path through an imagined matrix, the spatial system may only be engaged during encoding and retrieval. It is likely that during the retention interval the locations are imaged as a static pattern, because at this time they are not susceptible to interference from a spatial task (Quinn, 1991). They conclude 
that a spatial memory system is more likely to be involved when the order of locations must be retained. Smyth and Scholey further develop this idea by proposing that a sequence of locations is maintained using spatial attention.

One possibility is that what has been referred to as "spatial imagery", or perhaps one component of this system, is spatial attention, of the kind postulated by Smyth and Scholey, acting on representations supplied by the object system. It is not clear that spatial imagery will ultimately be reducible to spatial attention, although some investigators, most notably Kosslyn (e.g. Kosslyn et al., 1993), emphasise the attentional component in what is more generally referred to as spatial imagery. Whether one conceives of it as spatial imagery or spatial attention, what our results demonstrate is that it is specifically this system that aids in sequencing.

Manuscript received 3 July 1995 Manuscript accepted 15 September 1995

\section{REFERENCES}

Andrade, J., \& Meudell, P. (1993). Short report: Is spatial information encoded automatically in memory? The Quarterly Journal of Experimental Psychology, 46 A, 365-375.

Critchley, M. (1953). The parietal lobes. London: Edward Arnold.

Ellis, N.R. (1990). Is memory for spatial location automatically encoded? Memory \& Cognition, 18, 584-592.

Farah, M.J. (1989). The neuropsychology of mental imagery. In F. Boller \& J. Grafman (Eds.), Handbook of neuropsychology: Section 4. Disorders of visual behavior (pp.395-418). Amsterdam: Elsevier.

Farah, M.J., Hammond, K.M., Levine, D.N., \& Calvanio, R. (1988). Visual and spatial mental imagery: Dissociable systems of representation. Cognitive Psychology, 20, 439-462.

Friedman, W.J. (1983). Image and verbal processes in reasoning about the months of the year. Journal of Experimental Psychology: Learning, Memory, and Cognition, 9, 650-666.

Hasher, L., \& Zacks, R.T. (1979). Automatic and effortful processes in memory. Journal of Experimental Psychology: General, 108, 356-388.

Healy, A.F. (1974). Separating item from order information in short-term memory. Journal of Verbal Learning \& Verbal Behavior, 13, 644-655.

Healy, A.F. (1977). Pattern coding of spatial order information in short-term memory. Journal of Verbal Learning and Verbal Behavior, 16, 419-437.

Held, R. (1968). Dissociation of visual functions by deprivation and rearrangement. Psychologische Forschung, 31, 338-348.

Hitch, G., \& Morton, J. (1975). The unimportance of explicit spatial information in serial recall of visually presented lists. Quarterly Journal of Experimental Psychology, 27, 161-164.

Ingle, D. (1967). Two visual mechanisms underlying the behavior of fish. Psychologische Forschung, 31, 44-51.

Kosslyn, S.M., Alpert, N.M., Thompson, W.L., Maljkovic, V., Weise, S.B., Chabris, C.F., Hamilton, S.E., Rauch, S.L. \& Buonanno, F.S. (1993). Visual mental imagery activates topographically organized visual cortex: PET investigations. Journal of Cognitive Neuroscience, 5, 263-287.

Levine, D.N., Warach, J., \& Farah, M. (1985). Two visual systems in mental imagery: Dissociation of "what" and "where" in imagery disorders due to bilateral posterior cerebral lesions. Neurology, 35, 1010-1018.

Logie, R.H. (1995). Visuo-spatial working memory. Hove, UK: Lawrence Erlbaum Associates Ltd. 
